ARTICLE

Received 23 Aug 2016 | Accepted 24 Apr 2017 | Published 9 Jun $2017 \quad$ DOl: 10.1038/ncomms15767

\title{
Pumping approximately integrable systems
}

\author{
Florian Lange ${ }^{1}$, Zala Lenarčič ${ }^{1} \&$ Achim Rosch $^{1}$
}

Weak perturbations can drive an interacting many-particle system far from its initial equilibrium state if one is able to pump into degrees of freedom approximately protected by conservation laws. This concept has for example been used to realize Bose-Einstein condensates of photons, magnons and excitons. Integrable quantum systems, like the one-dimensional Heisenberg model, are characterized by an infinite set of conservation laws. Here, we develop a theory of weakly driven integrable systems and show that pumping can induce large spin or heat currents even in the presence of integrability breaking perturbations, since it activates local and quasi-local approximate conserved quantities. The resulting steady state is qualitatively captured by a truncated generalized Gibbs ensemble with Lagrange parameters that depend on the structure but not on the overall amplitude of perturbations nor the initial state. We suggest to use spin-chain materials driven by terahertz radiation to realize integrability-based spin and heat pumps.

\footnotetext{
${ }^{1}$ Institute for Theoretical Physics, University of Cologne, Zülpicher Straße 77a, D-50937 Cologne, Germany. Correspondence and requests for materials should be addressed to Z.L. (email: zala.lenarcic@thp.uni-koeln.de).
} 
A simple classical example for a weakly driven system is a well-insulated greenhouse. Due to the approximate conservation of the energy within the greenhouse, even weak sunlight can lead to high temperatures in its interior, which can be computed from the simple rate equation for the energy transfer. Similarly, large spin accumulation can be achieved in systems with approximate spin conservation ${ }^{1}$. Using approximate conservation of the number of photons, magnons or exciton polaritons, one can use pumping by light to reach densities, which allow for the realization of Bose-Einstein condensates ${ }^{2-4}$. Number-conserving collisions induce a quasi-equilibrium state in these systems, which can be efficiently described by introducing a chemical potential whose value is determined by balancing pumping and decay processes. Related theoretical approaches that describe electron-phonon systems far from equilibrium are so-called two-temperature models ${ }^{5}$ : here one uses that the energy of the electrons and phonons are approximately separately conserved to introduce two different temperatures for the subsystems.

Integrable many-particle systems, like the one-dimensional (1D) fermionic Hubbard model or the XXZ Heisenberg model, are described by an infinite number of (local or quasi-local) conservation laws $^{6-11}$. In closed integrable systems those prevent the equilibration into a simple thermal state, for example, after a sudden change of parameters. Instead the system can be described by a generalized Gibbs ensemble (GGE) ${ }^{12-21}$

$$
\rho_{0} \sim \exp \left(-\sum_{i} \lambda_{i} C_{i}\right)
$$

where $C_{i}$ are the conserved quantities and $\lambda_{i}$ the corresponding Lagrange parameters. It has also been shown experimentally ${ }^{22}$ that GGEs for a Lieb-Liniger model can provide highly accurate descriptions of interacting bosons in 1D.

Many materials are described with high accuracy by integrable models ${ }^{23}$, however, weak integrability breaking terms and the coupling to thermal phonons imply that in equilibrium these systems are described by simple thermal states, $\rho_{0} \sim \mathrm{e}^{-\beta H}$, instead of GGEs. The proximity to the integrable point and the presence of approximate conservation laws leads to enhanced spin or heat conductivities (within linear-response theory) ${ }^{24-26}$ and also to a slow relaxation after a quantum quench (via GGE-prethermalization) towards the equilibrium state ${ }^{27}$.

We will show that-as in the greenhouse example, see Fig. 1-such an approximately integrable system can be driven far from its thermal equilibrium by weak perturbations arising, for example, from a driving periodic in time or from coupling to a non-thermal bath. To balance the constant heating due to driving the system has to be weakly open, for example, by coupling to a phonon bath. As we will demonstrate this mechanism can be used for example to create large spin and heat currents. Besides the quasi-1D systems considered by us, also approximately many-body localized systems are characterized by infinitely many approximate conservation laws which may lead to a strong response to driving ${ }^{28,29}$.

\section{Results}

Weakly driven system. We consider an interacting many-body system that is approximately described by Hamiltonian $H_{0}$ and characterized by a finite or infinite number of (quasi-)local conserved quantities $C_{i},\left[H_{0}, C_{i}\right]=0$, one of them being $H_{0}$. Energy and other conservations are weakly broken by coupling to thermal or non-thermal baths and/or perturbations periodic in time. For simplicity, we assume periodic boundary conditions and a (discreet) translational invariance. We describe the system with density matrix $\rho$ whose dynamics is governed by the Liouvillian super-operator $\hat{\mathcal{L}}$,

$$
\dot{\rho}=\hat{\mathcal{L}} \rho, \quad \hat{\mathcal{L}}=\hat{\mathcal{L}}_{0}+\epsilon \hat{\mathcal{L}}_{1},
$$

where $\hat{\mathcal{L}}$ can be split into the dominant unitary Hamiltonian evolution $\hat{\mathcal{L}}_{0} \rho=-i\left[H_{0}, \rho\right]$ and perturbation $\hat{\mathcal{L}}_{1}$ of strength $\epsilon$. We are interested in the limit of small $\epsilon$ for $t \rightarrow \infty$, where a unique (Floquet) steady state $\rho_{\infty}$ is obtained. The general structure of perturbation theory in this case has, for example, been discussed in refs $30-32$. In this limit, $\rho_{\infty}$ can be approximated by $\rho_{0}=\lim _{\epsilon \rightarrow 0} \lim _{t \rightarrow \infty} \rho$ with $\hat{\mathcal{L}}_{0} \rho_{0}=0$ according to equation (2). We assume and later support numerically that $\rho_{0}$ is approximately described by a GGE, see equation (1).

Here it is essential to note that-as in the greenhouse example discussed above-the parameters $\lambda_{i}$ are not determined by the initial state but by the form of the weak perturbations $\hat{\mathcal{L}}_{1}$. Our central goal is to compute the $\lambda_{i}$. We first discuss the case of Lindblad dynamics, where perturbation theory linear in $\epsilon$ can be used, and then focus on Hamiltonian dynamics where we have to consider $\epsilon^{2}$ contributions.

Markovian perturbation. Within the Markovian approximation one can use the Lindblad form for $\hat{\mathcal{L}}_{1}$ (ref. 33). Note that Lindblad dynamics is considered here mainly for pedagogical purposes (formulas are simpler) while no Lindblad approximation is used for the models studied below. The coefficients $\lambda_{i}$ that fix the GGE are determined from the condition that the change of the approximately conserved quantities has to vanish in the steady state

$$
\left\langle\dot{C}_{i}\right\rangle=\operatorname{Tr}\left(C_{i} \hat{\mathcal{L}} \rho_{0}\right)=\operatorname{Tr}\left(C_{i} \epsilon \hat{\mathcal{L}}_{1} \rho_{0}\right) \stackrel{!}{=} 0,
$$

where we used that $\hat{\mathcal{L}}_{0} \rho_{0}=-i\left[H_{0}, \rho_{0}\right]=0$. Relation (3) yields a set of coupled equations for $\lambda_{i}$, where the number of equations is equal to the number of conserved quantities. We define the super-projector $\hat{P}$ onto the tangential space of GGE density matrix,

$$
\hat{P} X \equiv-\sum_{i, i^{\prime}} \frac{\partial \rho_{0}}{\partial \lambda_{i}}\left(\chi^{-1}\right)_{i i^{\prime}} \operatorname{Tr}\left(C_{i^{\prime}} X\right),
$$

using $\chi_{i i^{\prime}}=-\operatorname{Tr}\left(C_{i} \partial \rho_{0} / \partial \lambda_{i^{\prime}}\right)$. Then the conditions for $\rho_{0}$ can be compactly written as

$$
\hat{\mathcal{L}}_{0} \rho_{0}=0, \quad \hat{P}\left(\hat{\mathcal{L}}_{1} \rho_{0}\right)=0 .
$$

This equation can also be derived by considering higher order perturbations in $\epsilon$, see Methods for details.

Hamiltonian perturbation. For Hamiltonian dynamics $\epsilon \hat{\mathcal{L}}_{1} \rho=-i\left[H_{1}, \rho\right]$, where $H_{1}$ may be a sum of several integrability breaking perturbations. Perturbation theory linear in $\epsilon$ vanishes, $\operatorname{Tr}\left(C_{i} \epsilon \hat{\mathcal{L}}_{1} \rho_{0}\right)=0$ for all $\lambda_{i}$. Therefore one has to expand to order $\epsilon^{2}$ and equation (5) is replaced by

$$
\hat{\mathcal{L}}_{0} \rho_{0}=0, \quad \hat{P}\left(\hat{\mathcal{L}}_{1} \hat{\mathcal{L}}_{0}^{-1} \hat{\mathcal{L}}_{1} \rho_{0}\right)=0 .
$$

Since $\hat{P}\left(\hat{\mathcal{L}}_{1} \rho_{0}\right)=0, \quad \hat{\mathcal{L}}_{1} \rho_{0}$ is not in the kernel of $\hat{\mathcal{L}}_{0}^{-1}$. For periodic driving this equation has to be interpreted within the Floquet formalism, see Methods.

Model. As discussed in the introduction, our goal is to describe a situation which can be realized experimentally in spin-chain materials driven by lasers operating in the terahertz regime. We assume that spin chains are approximately described by a spin-1/2 XXZ Heisenberg model, possibly in the presence of an 
external magnetic field $B$,

$$
H_{0}=\sum_{j} \frac{J}{2}\left(S_{j}^{+} S_{j+1}^{-}+S_{j}^{-} S_{j+1}^{+}\right)+\Delta S_{j}^{z} S_{j+1}^{z}-B S_{j}^{z} .
$$

The system is driven out of equilibrium by a weak (integrability breaking) time-dependent perturbation

$$
H_{\mathrm{d}}=\epsilon_{\mathrm{d}} J \sum_{j}\left((-1)^{j+1} \mathbf{S}_{j} \cdot \mathbf{S}_{j+1} \sin (\omega t)+(-1)^{j} S_{j}^{z} \cos (\omega t)\right)
$$

with driving frequency $\omega$. This specific term has been chosen because it can induce heat and spin currents (as can be shown by a symmetry analysis), and because it can be realized experimentally. Such staggered exchange couplings and staggered magnetic fields arise naturally in certain compounds with (at least) two magnetic atoms per unit cell when coupled to uniform electric and magnetic fields, respectively ${ }^{34-37}$. See Fig. 1 for a schematic drawing of such a compound and Methods for concrete experimental suggestions. Therefore $H_{\mathrm{d}}$ can be realized by shining a laser (typically at terahertz frequencies) onto the sample. In this case $\epsilon_{\mathrm{d}}^{2}$ is proportional to the laser power. Note that for $T=0$ and $B=0$ in the adiabatic limit, $\omega \rightarrow 0$, equations (7) and (8) realize an adiabatic Thouless pump, where per pumping cycle one spin is transported by one unit cell ${ }^{38}$. We will be interested in the opposite regime of large $\omega$ and large (effective) temperatures.

Formally, the periodic perturbation $H_{\mathrm{d}}$ would drive the system to infinite temperature ${ }^{39-42}$ (up to remaining conservation laws ${ }^{43}$, possibly through a prethermal-like regime ${ }^{44}$ ). In a solid state experiment this is prohibited by the coupling to phonons and, ultimately, to the thermal environment of the experimental set-up. We mimic this effect by coupling the spin system to a bath of Einstein phonons, $H_{0}^{\mathrm{ph}}=\omega_{\mathrm{ph}} \sum_{j} a_{j}^{\dagger} a_{j}+\ldots$, where dots stand for the couplings to further reservoirs which guarantee that the phonon system is kept at fixed temperature $T_{\mathrm{ph}}, \rho_{\mathrm{ph}} \sim \mathrm{e}^{-H_{0}^{\mathrm{ph}} / T_{\mathrm{ph}}}$. See Methods for details on finite size calculation using a broadened distribution of phonon energies. The (weak) coupling to the spin system is described by

$$
\begin{aligned}
H_{\mathrm{ph}}=\epsilon_{\mathrm{ph}} J & \sum_{j}\left(\mathbf{S}_{j} \cdot \mathbf{S}_{j+1}\left(a_{j}+a_{j}^{\dagger}\right)\right. \\
& \left.+\gamma_{\mathrm{m}}\left(S_{j}^{x} S_{j+1}^{z}+S_{j}^{z} S_{j+1}^{x}\right)\left(a_{j}+a_{j}^{\dagger}\right)\right) .
\end{aligned}
$$

To obtain a unique steady state it is essential to break all symmetries, including the $S^{z}$ conservation. Relativistic effects which relax $S^{z}$ are mimicked by $\gamma_{\mathrm{m}}$ in our approach. We expect $\gamma_{\mathrm{m}} \ll 1$ in materials without heavy elements. For simplicity, we set $\gamma_{\mathrm{m}}=1$ within our numerics as this is found to minimize finite size effects, without a qualitative influence on the results. Besides phonons also other integrability breaking perturbations exist in real materials, including defects, which typically dominate at the lowest temperatures. For high temperatures of the order of $J$ (relevant for the considered set-up) it is realistic to assume that phonon coupling dominates.

In the presence of a periodic perturbation, equation (8), in the long-time limit the density matrix is changing periodically, $\rho(t \rightarrow \infty)=\sum_{n} \mathrm{e}^{-i \omega n t} \rho^{(n)}$ with $\rho^{(n)^{\dagger}}=\rho^{(-n)}, n \in \mathbb{Z}$. Within the Floquet formalism one therefore promotes the steady-state density matrix to a vector and Liouville operator to a matrix, see Methods. For weak driving, $\epsilon_{\mathrm{d}} \rightarrow 0$, only the $n=0$ sector remains and the GGE ansatz, equation (1), simply reads $\rho_{0}^{(n)}=\left(\rho_{0} \otimes \rho_{\mathrm{ph}}\right) \delta_{n, 0}$, where we included also the phonon density matrix, see above.
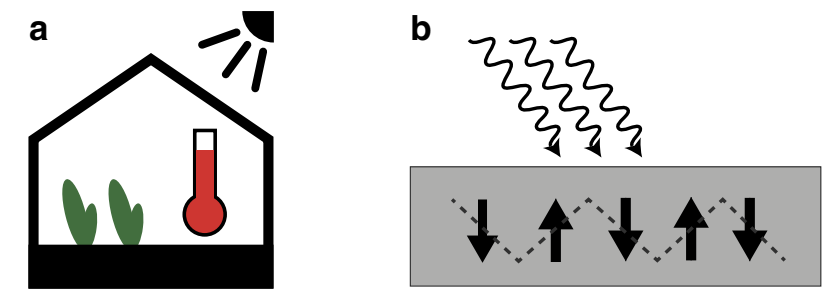

Figure 1 | Greenhouse principle. (a) A well-insulated greenhouse exposed to sunshine can heat up significantly since energy within it is approximately conserved. (b) As the heat current in spin-chain materials is approximately conserved even weak terahertz radiation can induce large heat current.

Material candidates must have appropriate crystal structure, schematically denoted by dashed lines indicating alternating chemical bonds.

Steady state. We will use two different approaches to determine an approximate solution for the steady-state density matrix. First, we will parametrize $\rho_{0}$, equation (1), with a small number of (quasi-)local conserved quantities, $C_{i}, i=1, \ldots, N_{\mathrm{C}}$. In an alternative approach, feasible for small systems, we take all conserved quantities into account: local and non-local, commuting and non-commuting. While the second approach is formally exact in the limit $\epsilon_{\mathrm{d}}, \epsilon_{\mathrm{ph}} \rightarrow 0$, the first one is, perhaps, more intuitive and can be computed for larger system sizes.

For the XXZ Heisenberg model an infinite set of mutually commuting local conserved quantities $C_{i}$ is known, see Methods. $C_{1}$ is the total spin $C_{1}=\sum_{i} S_{i}^{z}$ and $C_{2}=H_{\mathrm{XxZ}}$. Importantly, $C_{3}$ is the heat current ${ }^{45} C_{3}=J_{\mathrm{H}}(B=0)$. In addition there also exist (infinite) sets of quasi-local commuting conserved quantities ${ }^{8-10}$. As shown in refs 8,46 the spin-reversal parity-odd family has an overlap with the spin current $J_{S}$ at $\Delta<J$. Therefore both heat and spin current could show a large response to a weak perturbation. For our analysis, we choose three or five $\left(N_{\mathrm{C}}=4, N_{\mathrm{C}}=6\right)$ most local conserved quantities $C_{i}, i=1, \ldots, N_{\mathrm{C}}-1$. From the quasi-local sets, we include as a single (effective) operator the conserved part of spin current $J_{S}^{c}$, computed numerically ${ }^{25,47}$. For details see Methods. In the presence of an external magnetic field, equation (7), the heat current also has, in addition to $C_{3}$, a spin current component, $J_{\mathrm{H}}=C_{3}-B J_{\mathrm{S}}$ (ref. 45 ).

For the visualization of our results it is useful to define generalized forces $F_{i}$ in the space of Lagrange parameters by rewriting $\hat{P} \dot{\rho}=\sum_{i} \frac{\partial \rho_{0}}{\partial \lambda_{i}} F_{i}$ such that $\dot{\lambda}_{i} \approx F_{i}$,

$$
F_{i}=\sum_{i^{\prime}}\left(\chi^{-1}\right)_{i i^{\prime}} \operatorname{Tr}\left(C_{i^{\prime}} \epsilon \hat{\mathcal{L}}_{1} \hat{\mathcal{L}}_{0}^{-1} \epsilon \hat{\mathcal{L}}_{1} \rho_{0}\right)
$$

computed using exact diagonalization, see Methods. The vector $\mathbf{F}$ is a function of the Lagrange parameters $\lambda_{i}$, which points into the direction of the steady-state stable fixed point obtained from $F_{i}=0$. In the absence of driving (Fig. 2a) one obtains the expected thermal state with $T=T_{\mathrm{ph}}$ while all other Lagrange parameters $\lambda_{i}$ vanish. For finite driving the GGE is activated and the $\lambda_{i}$ become finite (Fig. 2b). To obtain the steady state, we solve $\chi \mathbf{F}=0$ using Newton's method.

For the second approach, performed on small $\mathrm{N}$-site systems, we first numerically construct a basis in the set of all (local and non-local) conserved operators, $\mathcal{Q}=\left\{|n\rangle\langle m|\right.$, with $\left.E_{m}^{0}=E_{n}^{0}\right\}$, where $H_{0}|n\rangle=E_{n}^{0}|n\rangle$. Due to degeneracies we find (for finite $B$ and $\Delta \neq J$ ) about $2 \cdot 2^{N}$ elements $Q_{i} \in \mathcal{Q}$. In the limit $\epsilon_{\mathrm{d}}, \epsilon_{\mathrm{ph}} \rightarrow 0$ the steady-state density matrix $\rho_{\infty}$ has to fulfil $\mathcal{L}_{0} \rho_{\infty}=0$ and therefore can be exactly written as a linear combination of the $Q_{i}$, $\rho_{\infty}=\sum \alpha_{i} Q_{i}$. Using equation (6), we therefore find that the steady-state density matrix for $\epsilon_{\mathrm{d}}, \epsilon_{\mathrm{ph}} \rightarrow 0$ is exactly given by the unique eigenvector with eigenvalue zero of the matrix

$$
\mathcal{L}_{m n}^{\mathcal{Q}}=-\operatorname{Tr}\left(Q_{m}^{\dagger} \epsilon \hat{\mathcal{L}}_{1} \hat{\mathcal{L}}_{0}^{-1} \epsilon \hat{\mathcal{L}}_{1} Q_{n}\right),
$$



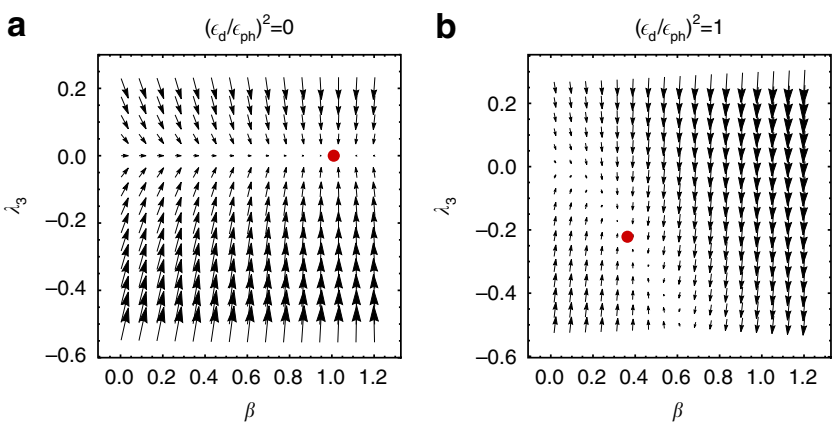

Figure 2 | Force field with thermal and non-thermal steady state. Effective force $\mathbf{F}$ in the space of Lagrange parameters $\left(\beta, \lambda_{3}\right)$ using $e^{-\beta H_{0}-\lambda_{3} C_{3}}$ as an ansatz for the generalized Gibbs ensemble. Parameters: $J=\Delta=-B=\omega=\omega_{\mathrm{ph}}=T_{\mathrm{ph}}$. Lagrange parameters $\left(\beta, \lambda_{3}\right)$ are plotted in units $1 / J$ and $1 / \mathcal{J}^{2}$, respectively. (a) In the absence of an external driving, $\epsilon_{\mathrm{d}}=0$, the stable fixed point (red dot) is given by the thermal ensemble, $\beta=1 / T_{\mathrm{ph}}, \lambda_{3}=0$. (b) When the system is driven by $H_{\mathrm{d}}\left(\epsilon_{\mathrm{d}}=\epsilon_{\mathrm{ph}}\right)$, it heats up and $\lambda_{3}$ becomes finite.
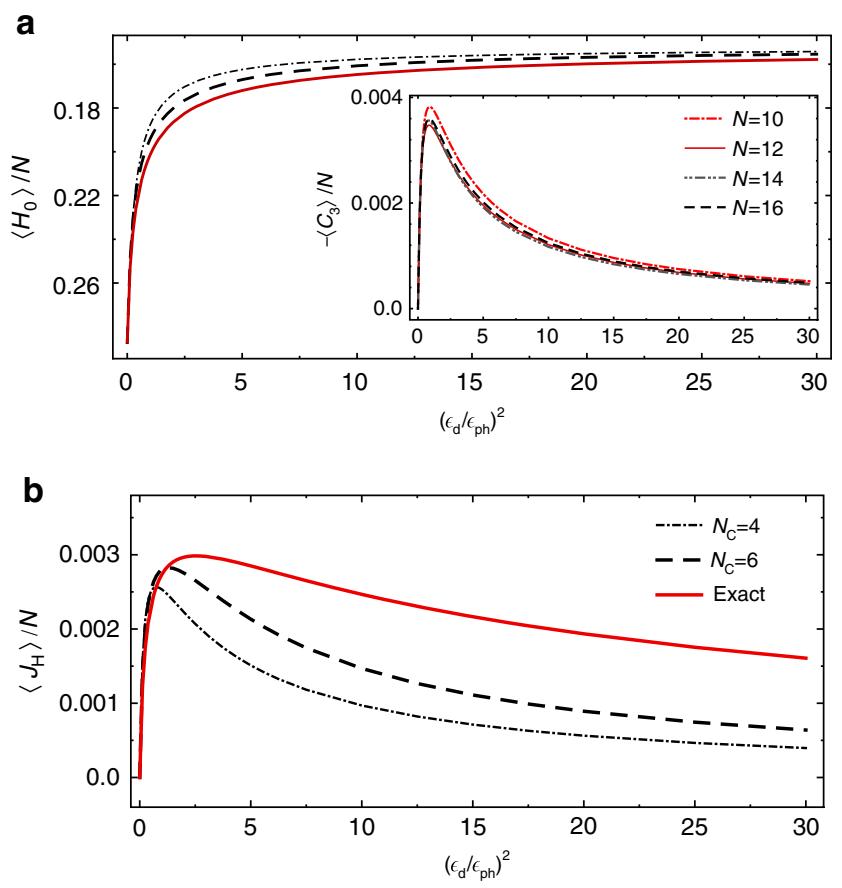

Figure 3 | Steady-state expectation values of conserved quantities. Expectation values of (a) energy and (b) heat current densities for a weakly driven spin chain, $\epsilon_{\mathrm{d}}, \epsilon_{\mathrm{ph}} \rightarrow 0$, as functions of the ratio of driving strength $\epsilon_{\mathrm{d}}$ and phonon coupling $\epsilon_{\mathrm{ph}}$. Red solid lines: exact result taking into account all 7,969 conservation laws of a system of $N=12$ sites.

(a) For the energy accurate results are already obtained with a GGE ensemble based on $N_{C}=4$ (dot-dashed lines) or $N_{C}=6$ (dashed lines) conserved quantities. (b) Also the heat current $J_{H}=C_{3}-B J_{S}$ is qualitatively well described by the GGE ensemble but quantitative deviations are larger. Inset: Finite size analysis for (local) $C_{3}$ based on GGE ensemble with $N_{C}=6$ conserved quantities. Parameters: $J=1$, $\Delta=0.8, B=-1.0, \omega=1.6 \omega_{\mathrm{ph}}, \omega_{\mathrm{ph}}=T_{\mathrm{ph}}=1$.

where $\hat{\mathcal{L}}_{1}, \hat{\mathcal{L}}_{0}$ are Floquet matrices, see Methods. Note that only the relative $\epsilon_{\mathrm{d}} / \epsilon_{\mathrm{ph}}$ and not the absolute strength of perturbations determine $\rho_{0}$, as can be seen by dividing the equations $\chi \mathbf{F}=0$ or $\mathcal{L}^{\mathcal{Q}} \rho_{0}=0$ by $\epsilon_{\text {ph }}^{2}$.

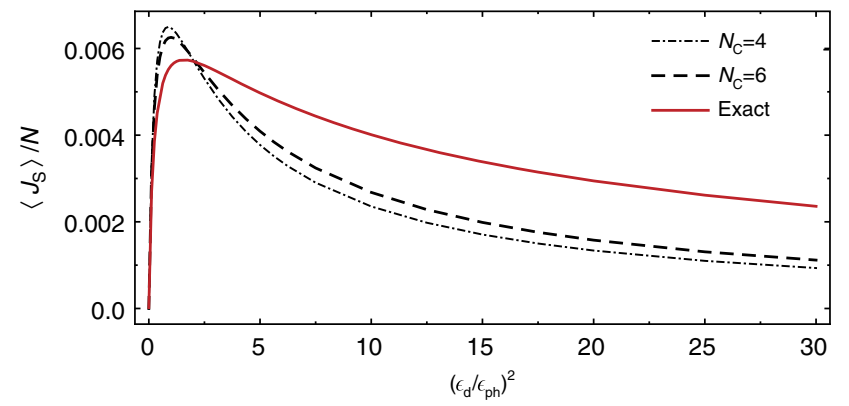

Figure 4 | Spin current in the steady state. For vanishing magnetic field a spin current (but no heat current) is generated within our model for finite ratios of $\epsilon_{\mathrm{d}} / \epsilon_{\mathrm{ph}}$. The expectation value of spin current density is again maximal for $\epsilon_{\mathrm{d}} / \epsilon_{\mathrm{ph}} \approx 1$. Parameters: $J=1, \Delta=0.8, \omega=1.6 \omega_{\mathrm{ph}}, \omega_{\mathrm{ph}}=T_{\mathrm{ph}}=1, N=12$.

In Fig. 3, we show the expectation value of the energy and of the heat current densities as functions of $\epsilon_{\mathrm{d}} / \epsilon_{\mathrm{ph}}$ taking into account $N_{\mathrm{C}}=4, N_{\mathrm{C}}=6$, and all conserved quantities. The energy density expectation value is already obtained with good accuracy for $N_{\mathrm{C}}=4$ and even better for $N_{\mathrm{C}}=6$. The heat current vanishes both in thermal equilibrium, $\epsilon_{\mathrm{d}} \rightarrow 0$, and for $\epsilon_{\mathrm{ph}} \rightarrow 0$, where the system is described by an infinite temperature state with finite magnetization, $\rho_{0} \sim \mathrm{e}^{-\lambda_{1} S^{z}}$ and $\left\langle H_{0}\right\rangle=-B\left\langle S_{z}\right\rangle$. It takes its largest value for $\epsilon_{\mathrm{ph}} \sim \epsilon_{\mathrm{d}}$. For the currents a description in terms of $N_{\mathrm{C}}=4$ or 6 is qualitatively but not quantitatively accurate. Our study strongly suggests that further quasi-local conserved quantities contribute, as discussed in quench protocols ${ }^{15-17}$, see also ref. 25. For the chosen parameters our results depend only weakly on the system size $N$, see inset of Fig. 3 . System size analysis is performed for $N_{\mathrm{C}}=6$ since the solution based on all conservations cannot be obtained for larger systems.

Our set-up can also be used to create spin currents. Whilst, by symmetry (bond-centered rotation in real and spin space by $\pi$ around $y$ axis), a finite external field $B$ is needed to obtain a finite heat current, this is not the case for the spin current. Figure 4 displays the spin current density as a function of $\epsilon_{\mathrm{d}} / \epsilon_{\mathrm{ph}}$ for $B=0$. Qualitatively one obtains a behaviour rather similar to the results for the heat current shown in Fig. 3 with a maximum in the spin current for $\epsilon_{\mathrm{ph}} \sim \epsilon_{\mathrm{d}}$.

The external magnetic field $B$ is a parameter which can easily be tuned experimentally. Figure 5 shows heat and spin current densities as a function of external magnetic field $B$ for $\left(\epsilon_{\mathrm{d}} / \epsilon_{\mathrm{ph}}\right)^{2}=2.5$. Note that the sign of the magnetic field determines the sign of the heat current $\left\langle J_{\mathrm{H}}\right\rangle=\left\langle C_{3}\right\rangle-B\left\langle J_{\mathrm{S}}\right\rangle$. All main features of the B-dependence are semi-quantitatively reproduced by the truncated GGE with $N_{\mathrm{C}}=6$. For very large magnetic fields the convergence to the steady-state fixed point becomes slow as transitions rates connecting sectors with different magnetization are strongly suppressed, see Methods for further details.

\section{Discussion}

We have demonstrated that driving approximately integrable systems activates and pumps into approximately conserved quantities. Perhaps the most simple experimental set-up to measure the pumping effect predicted in this work, is to use a terahertz laser that excites a spin-chain material like Cu-benzoate where by symmetry staggered terms of the form (8) are expected $^{34,35}$. As a consequence of the induced heat currents it is anticipated that the system cools down on one side while it heats upon the other. The direction of the effect can be controlled 

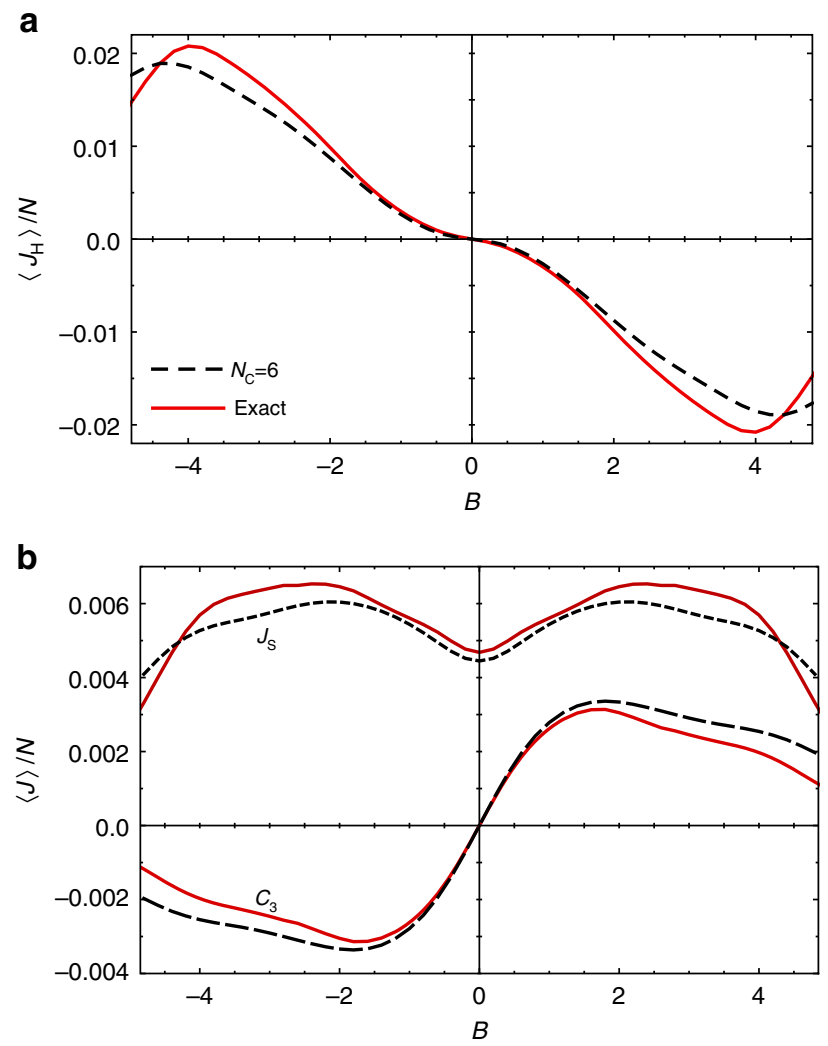

Figure 5 | Currents in the steady state at different magnetic field.

(a) Heat current $J_{\mathrm{H}^{\prime}}$ (b) spin current $J_{\mathrm{S}}$ and $C_{3}$ densities as a function of external magnetic field $B$ obtained from a GGE ensemble with $N_{C}=6$ conserved quantities (dashed) or from an exact calculation (solid) including all conservations. Parameters: $\left(\epsilon_{\mathrm{d}} / \epsilon_{\mathrm{ph}}\right)^{2}=2.5, J=1, \Delta=0.8$, $\omega=1.6 \omega_{\mathrm{ph}}, \omega_{\mathrm{ph}}=T_{\mathrm{ph}}=1, N=12$.

either by changing the direction of the laser beam or the sign of the external magnetic field $B$.

For the chosen parameters the spin and heat currents expressed in dimensionless units appear to be rather small, of the order of $10^{-3}$. While these values can definitely be increased by tuning parameters, for example the external magnetic field, it is important to note that the currents are actually quite large compared to the typical heat or spin currents obtained in bulk materials. To create a heat current of similar size in a good heat conductor like $\mathrm{Cu}$ (assuming $J \sim k_{\mathrm{B}} \cdot 100 \mathrm{~K}, 5 \AA$ for the distance of the spin chains, and $\kappa^{\mathrm{Cu}} \approx 400 \mathrm{Wm}^{-1} \mathrm{~K}^{-1}$ ) one would need a temperature gradient of several $10^{5} \mathrm{Km}^{-1}$. Similarly, to create a (transversal) spin current of comparable size in a heavy element like Pt using the spin Hall effect (assuming $\rho^{\mathrm{Pt}} \approx 10 \mu \Omega \mathrm{cm}$ and $\alpha_{\mathrm{s}}^{\mathrm{Pt}} \approx 10 \%$ for the spin Hall angle ${ }^{48}$ ) one needs electric fields of the order of $10^{4} \mathrm{Vm}^{-1}$ or sizable current densities of the order of $10^{11} \mathrm{Am}^{-2}$. These numbers are even more remarkable when one takes into account that the electron densities in $\mathrm{Cu}$ or Pt are at least an order of magnitude higher than the spin density for spin chains with a distance of $5 \AA$.

While our study has focused on the steady state, it is instructive to discuss the relevant timescales for its buildup. For this argument, we consider a quench where at time $t=0$ an initial state is perturbed both by the integrable part of the Hamiltonian and by small non-integrable perturbations. At short times of the order of several $1 / J$ the initial state will prethermalize ${ }^{27,49-52}$ into a GGE, where the values of the conserved quantities, $\left\langle C_{i}\right\rangle$, are set by the initial conditions (with small corrections from the perturbations ${ }^{52,53}$ ). Further time evolution can be approximately described by a GGE with time-dependent Lagrange parameters. Their time-dependence is determined by perturbations which assert forces $F_{i} \sim \epsilon^{2}$, such that $\mathrm{d} \lambda_{i} / \mathrm{d} t \approx F_{i}$. Governed by the perturbations the system will loose the memory of its initial condition on a timescale of order $1 / \epsilon^{2}$ and relax to the steady state (obtained from $F_{i}=0$ ) which is, in general, completely unrelated to the prethermalized state. Note that the same approach predicts ordinary thermalization in the absence of external driving.

Our results suggest that the concept of generalized GGEs has a much broader range of application than previously anticipated, now extended to open systems where symmetries are not exact and integrability is weakly broken. A truncated GGE proved to be useful for qualitative description, however, it showed quantitative discrepancies most probably due to disregarded quasi-local conserved quantities, as observed already in quench protocols ${ }^{15,16}$. We are planning a future study tailored to address this issue systematically. It would be interesting to develop integrability-based methods similar to the quench-action approach $^{15,16,54,55}$ to treat such situations.

Most important for applications is that the integrability is not required to be realized exactly but only approximately. Efficient pumping requires only that the pumping rates are of the same order of magnitude as the loss rates arising from integrability breaking terms. Especially the integrability-based creation of large spin currents could find its application in future spintronics devices.

\section{Methods}

Perturbing around $\rho_{\mathbf{0}}$. The central equations (5) or (6), used to determine the density matrix $\rho_{0}$ in the limit $\epsilon \rightarrow 0$, have to be consistent and can also even be derived by considering perturbations around $\rho_{0}, \rho_{\infty}=\rho_{0}+\delta \rho$.

First, the leading $\delta \rho$ correction to $\left\langle\dot{C}_{i}\right\rangle$, equation (3), arising from $\operatorname{Tr}\left(C_{i} \hat{\mathcal{L}}_{0} \delta \rho\right)$ which is nominally of the same order as $\operatorname{Tr}\left(C_{i} \epsilon \hat{\mathcal{L}}_{1} \rho_{0}\right)$ vanishes trivially as $\operatorname{Tr}\left(C_{i}\left[H_{0}, \delta \rho\right]\right)=\operatorname{Tr}\left(\delta \rho\left[C_{i}, H_{0}\right]\right)=0$.

For arbitrary $\rho_{0}, \delta \rho$ is exactly given by $\delta \rho=-\hat{\mathcal{L}}^{-1} \epsilon \hat{\mathcal{L}}_{1} \rho_{0}$, where $\hat{\mathcal{L}}^{-1}$ is a short-hand notation for $\lim _{\eta \rightarrow 0}(\hat{\mathcal{L}}-\eta \hat{1})^{-1}$ with the infinitesimal regularizer $\eta$. The correct expansion point $\rho_{0}$ is found if $\lim _{\epsilon \rightarrow 0} \delta \rho=0$. Below we show that for the projection operator $\hat{P}$, equation (4),

$$
\hat{\mathcal{L}}^{-1} \hat{P} \sim \mathcal{O}\left(\epsilon^{-1}\right),
$$

which would yield $\hat{\mathcal{L}}^{-1} \hat{P} \in \hat{\mathcal{L}}_{1} \rho_{0} \sim \mathcal{O}(1)$. This contradicts our perturbative approach unless $\hat{P} \hat{\mathcal{L}}_{1} \rho_{0}=0$, as set by our condition equation (5).

Equation (12) is a consequence of the fact that $\hat{P}$ projects onto the tangential space to GGE density matrix. In this space $\hat{\mathcal{L}}_{0}$ vanishes by definition,

$\hat{\mathcal{L}}_{0}\left(\partial \rho_{0} / \partial \lambda_{i}\right)=0$, and $\hat{\mathcal{L}}=\hat{\mathcal{L}}_{0}+\epsilon \hat{\mathcal{L}}_{1}$ is therefore of order $\epsilon$. Technically, this can be seen by using the general relation

$$
(\hat{X}+\hat{Y})^{-1}-\hat{X}^{-1}=-(\hat{X}+\hat{Y})^{-1} \hat{Y} \hat{X}^{-1},
$$

for

$$
\begin{aligned}
& \hat{X}=\hat{P}_{\epsilon} \hat{\mathcal{L}}_{1} \hat{P}, \\
& \hat{Y}=\hat{\mathcal{L}}_{0}+\hat{Q} \epsilon \hat{\mathcal{L}}_{1} \hat{Q}+\hat{P} \epsilon \hat{\mathcal{L}}_{1} \hat{Q}+\hat{Q} \epsilon \hat{\mathcal{L}}_{1} \hat{P},
\end{aligned}
$$

with $\hat{Q}=\hat{1}-\hat{P}$ and $\hat{X}+\hat{Y}=\hat{\mathcal{L}}$. Then

$$
\begin{aligned}
\hat{\mathcal{L}}^{-1} \hat{P} & =(\hat{X}+\hat{Y})^{-1} \hat{P} \\
& =\hat{X}^{-1} \hat{P}-(\hat{X}+\hat{Y})^{-1} \hat{Q} \hat{Y} \hat{P} \hat{X}^{-1} \hat{P} \\
& \sim \mathcal{O}\left(\epsilon^{-1}\right)+\mathcal{O}(1)
\end{aligned}
$$

The second term is $O(1)$ as $\hat{\mathcal{L}}_{0} \hat{P}=0$ and therefore $\hat{Y} \hat{P} \sim O(\epsilon)$. The divergence of $\hat{\mathcal{L}}^{-1} \hat{P}$ for $\epsilon \rightarrow 0$ can be directly related to the fact that integrable systems are characterized by infinite conductivities (finite Drude weights) at finite temperatures $^{56}$ as can, for example, be seen ${ }^{24}$ within the memory matrix formalism ${ }^{57}$.

All arguments given above can be generalized to situations where leading corrections arise from second-order perturbation theory in which case one obtains equation (6) instead of equation (5). 

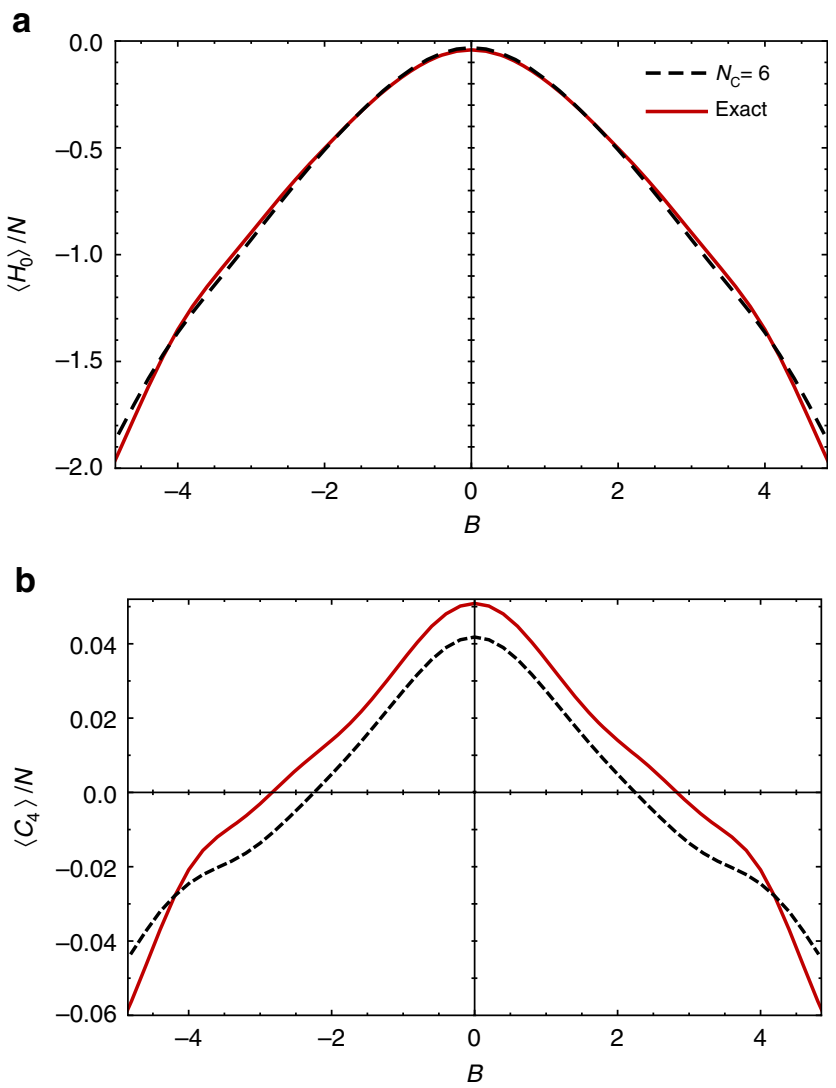

Figure 6 | Additional verification of generalized Gibbs ensembles.

(a) The energy density and (b) the expectation value of another conserved quantity $C_{4}$ (4-spin operator) as a function of magnetic field $B$, obtained from calculation using all conserved quantities (solid) and a GGE with $N_{\mathrm{C}}=6$ (quasi-) local conserved quantities (dashed).

Staggered hopping and magnetic field modulation. Sizable staggered g-tensors leading to staggered B-fields have been observed in a number of different compounds ${ }^{34-37}$. Similarly an external electric field will distort the crystalline structure in these materials, leading to staggered exchange couplings linear in homogeneous electric fields. An example of such a material is $\mathrm{Cu}$-benzoate ${ }^{34}$ with the above modulations allowed by symmetry for electric (magnetic) fields applied in the 010 (001) crystallographic direction. In this system the staggered g-tensor has been measured to be $\sim 0.08$ (ref. 35), the size of the staggered exchange coupling is unknown. For simplicity, we assume in equation (8) that the two staggered terms are of the same size.

Conservation laws of the XXZ Heisenberg model. An infinite set of local conserved quantities $C_{i}$ of the Heisenberg model $H_{\mathrm{Xxz}}=H_{0}(B=0)$ can be obtained using the boost operator $O_{\mathrm{b}}=-i \sum_{j} j h_{j, j+1}$ (where $H_{\mathrm{XXz}}=\sum_{j} h_{j, j+1}$ ) from the recursion relation $\left[O_{\mathrm{b}}, C_{i}\right]=C_{i+1}$ for $i>1$ with $C_{1}=\sum_{j} S_{j}^{z}, C_{2}=H_{\mathrm{Xxz}}$ (ref. 7). In general, $C_{i}$ are operators involving maximally $i$ neighbouring sites. Importantly, $C_{3}$ in the absence of external magnetic field equals the heat current

$$
J_{\mathrm{H}}(B=0)=C_{3}=J^{2} \sum_{j}\left(\mathbf{S}_{j}^{\prime} \times \mathbf{S}_{j+1}^{\prime \prime}\right) \cdot \mathbf{S}_{j+2}^{\prime}
$$

with rescaled spin operators $S_{j}^{\prime a}=\sqrt{\lambda_{a}} S_{j}^{a}, S_{j}^{\prime \prime a}=\sqrt{\lambda_{z} / \lambda_{a}} S_{j}^{a}$ for $\lambda_{z}=\Delta / J, \lambda_{x}=\lambda_{y}=1$. In the presence of external magnetic field, equation (7), heat current has in addition to $C_{3}$ also a spin current component,

$$
J_{\mathrm{H}}=J^{2} \sum_{j}\left(\mathbf{S}_{j}^{\prime} \times \mathbf{S}_{j+1}^{\prime \prime}\right) \cdot \mathbf{S}_{j+2}^{\prime}-B J_{\mathrm{S}}
$$

As understood recently there also exist families of quasi-local conserved quantities ${ }^{8-10}$, which are mostly disregarded in our study with the exception of a spin-reversal parity-odd operator, $J_{S}^{c}$. The latter is constructed as the conserved part of the spin current operator $J_{\mathrm{S}}$,

$$
\begin{aligned}
& J_{S}=i \frac{J}{2} \sum_{j}\left(S_{j}^{+} S_{j+1}^{-}-S_{j}^{-} S_{j+1}^{+}\right) \\
& J_{S}^{\mathrm{c}}=\sum_{\tilde{n}}|\tilde{n}\rangle\left\langle\tilde{n}\left|J_{S}\right| \tilde{n}\right\rangle\langle\tilde{n}|
\end{aligned}
$$

where $|\tilde{n}\rangle$ are simultaneous eigenstates of the $C_{i}$. Since it is known that the spin current has an overlap with the quasi-local family ${ }^{45}$ for $\Delta<J$, the conserved $J_{S}^{\complement}$ contains quasi-local components (and, possibly, non-local components not contributing in the thermodynamic limit).

Floquet formulation. For a periodically driven system described by $\dot{\rho}=\hat{\mathcal{L}}(t) \rho$ with $\hat{\mathcal{L}}(t+T)=\hat{\mathcal{L}}(t)$ the density matrix changes periodically in the long-time limit. Therefore it is useful to split it into Floquet components,

$$
\rho=\sum_{n} \mathrm{e}^{-i n \omega t} \rho^{(n)}, n \in \mathbb{Z}
$$

with $\rho^{(-n)}=\rho^{(n)^{\dagger}}$ and $\omega=2 \pi / T$. The Floquet components are combined into the vector $\rho=\left(\ldots \rho^{(-1)}, \rho^{(0)}, \rho^{(1)}, \ldots\right)$. The Liouvillian is promoted to a (static) matrix $\hat{\mathcal{L}}_{n m}=i n \omega \delta_{n m}+\hat{\mathcal{L}}_{n-m}$ with $\hat{\mathcal{L}}_{n-m}=\frac{1}{T} \int_{0}^{T} \hat{\mathcal{L}}(t) \mathrm{e}^{i \omega(n-m) t} \mathrm{~d} t$. Using this notation, all results obtained for static Liouvillian super-operators directly translate to the time-periodic case. Within our set-up $H_{0}$, all approximate conservation laws $C_{i}$ and the GGE density matrix $\rho_{0}$ are static and therefore the projection operator $\hat{P}$, equation (4), projects onto the $n=0$ Floquet sector only. The steady-state condition, equation (6), thus means that the approximately conserved quantities do not grow after averaging over an oscillation period. To second order in $\epsilon_{\mathrm{d}}$ only transitions from the $n=0$ to the $n= \pm 1$ Floquet sector and back contribute to equations (6) or (10) as $\hat{\mathcal{L}}_{n}=0$ for $|n|>1$.

For the generalized force due to the periodic driving, we obtain from (10)

$$
\begin{aligned}
& F_{i}^{(d)}=\frac{2 \pi}{N} \epsilon_{\mathrm{d}}^{2} \sum_{i^{\prime}}\left(\chi^{-1}\right)_{i i^{\prime}} \sum_{m, k} \rho_{m}\left(C_{i^{\prime}, m}-C_{i^{\prime}, k}\right) \\
& \times\left\{\left|\left\langle k\left|H_{\mathrm{d}}^{(+)}\right| m\right\rangle\right|^{2} \delta\left(E_{k}^{0}-E_{m}^{0}-\omega\right)+\left|\left\langle k\left|H_{\mathrm{d}}^{(-)}\right| m\right\rangle\right|^{2} \delta\left(E_{k}^{0}-E_{m}^{0}+\omega\right)\right\}
\end{aligned}
$$

where we used $H_{0}$ eigenstates $|m\rangle$ with $H_{0}|m\rangle=E_{m}^{0}|m\rangle$, matrix elements $\rho_{m}=\left\langle m\left|\rho_{0}\right| m\right\rangle, C_{i, m}=\left\langle m\left|C_{i}\right| m\right\rangle$, and the notation $H_{\mathrm{d}}=\epsilon_{\mathrm{d}}\left(\mathrm{e}^{i \omega t} H_{\mathrm{d}}^{(-)}+\mathrm{e}^{-i \omega t} H_{\mathrm{d}}^{(+)}\right)$. Note that equation (20) contains-as expected-transition rates well-known from Fermi's golden rule. Equation (20) is evaluated for finite systems of size $N$ by replacing the $\delta$ function by a Lorentzian $(1 / \pi) \eta /\left(\omega^{2}+\eta^{2}\right)(\eta=0.1 J$ for $N=12)$.

Equation (20) is only valid for situations where all conservation laws commute with each other, with $C_{i}=\sum_{m}|m\rangle C_{i, m}\langle m|$, see below for a brief discussion of the non-commuting case.

Phonon coupling. As written in the main text, we assume that the phonon system always remains at equilibrium, $\rho_{\mathrm{ph}} \sim \mathrm{e}^{-H_{0}^{\mathrm{p}} / T_{\mathrm{ph}}}$. Using equation (10), after tracing over phonons, we obtain for the generalized force

$$
\begin{aligned}
& F_{i}^{(\mathrm{ph})}=2 \pi \epsilon_{\mathrm{ph}}^{2} \sum_{i^{\prime}}\left(\chi^{-1}\right)_{i^{\prime}} \sum_{m, k} \rho_{m}\left(C_{i^{\prime}, m}-C_{i^{\prime}, k}\right) \\
& \times J^{2}\left(\left|\left\langle k\left|\mathbf{S}_{j} \cdot \mathbf{S}_{j+1}\right| m\right\rangle\right|^{2}+\gamma_{\mathrm{m}}^{2}\left|\left\langle k\left|S_{j}^{x} S_{j+1}^{z}+S_{j}^{z} S_{j+1}^{x}\right| m\right\rangle\right|^{2}\right) \\
& \times\left(\left(n_{\mathrm{B}}\left(E_{m}^{0}-E_{k}^{0}\right)+1\right) A^{(\mathrm{ph})}\left(E_{m}^{0}-E_{k}^{0}\right)+n_{\mathrm{B}}\left(E_{k}^{0}-E_{m}^{0}\right) A^{(\mathrm{ph})}\left(E_{k}^{0}-E_{m}^{0}\right)\right)
\end{aligned}
$$

where $n_{\mathrm{B}}(E)=1 /\left(\mathrm{e}^{E / T_{\mathrm{ph}}}-1\right)$ is the equilibrium Bose distribution evaluated at the temperature $T_{\mathrm{ph}}$ and $A^{(\mathrm{ph})}(\omega)$ is the phonon spectral function. For our finite size calculation, we broaden the spectral function of the Einstein phonons using $A^{(\mathrm{ph})}(\omega)=\Theta(\omega) \frac{\omega}{\omega_{\mathrm{ph}} \eta \sqrt{\pi}} \mathrm{e}^{-\left(\omega-\omega_{\mathrm{ph}}\right)^{2} / \eta^{2}}$. This choice of broadening ensures detailed balance relations (necessary to obtain a thermal state in the absence of driving) and the positivity of phonon frequencies (necessary for stability). For all plots we use $\eta=0.4 J$. However, we have checked that similar results are obtained, for example, for $\eta=0.1 \mathrm{~J}$ for magnetic fields up to $|B|=2 J$. For larger fields $\eta=0.1 \mathrm{~J}$ does not provide a sufficient amount of relaxation between sectors with different magnetization and convergence becomes slow and unstable. For $\eta=0.4 J$ larger fields, $|B| \lesssim 5 J$, can be reached.

Implementation of non-commuting conservation laws. As discussed in the main text, a complete basis of all non-local commuting or non-commuting conserved quantities is given by $\mathcal{Q}=\left\{|n\rangle\langle m|\right.$ with $\left.E_{m}^{0}=E_{n}^{0}\right\}$ which solve the equation $\hat{\mathcal{L}}_{0} Q_{i}=0$ for $Q_{i} \in \mathcal{Q}$. Using the exact eigenstates of $H_{0}$ it is straightforward to evaluate equation (11), where we use for our finite size calculations the broadening procedures described above. As a technical detail, we note that, when one follows this procedure, one has to evaluate in the phonon sectors integrals of the type $\int \frac{A^{(\text {ph })}\left(\omega^{\prime}\right)}{\omega-\omega^{\prime}} n_{\mathrm{B}}\left(\omega^{\prime}\right) \mathrm{d} \omega^{\prime}$ numerically. For efficient evaluations, we use interpolating functions for these integrals. 
GGE estimation for other conserved quantities. To provide further support for our claim that truncated GGEs give a semi-quantitative description of our weakly open system we show in Fig. 6 additional comparison of the $\left\langle H_{0}\right\rangle$ and $\left\langle C_{4}\right\rangle$ as a function of magnetic field $B$ at $\left(\epsilon_{\mathrm{d}} / \epsilon_{\mathrm{ph}}\right)^{2}=2.5$, comparing as in the main text the exact calculation including all conserved quantities and the truncated GGE with $N_{\mathrm{C}}=6$ (quasi-)local conserved quantities. The GGE ansatz captures the right magnitude and the correct behaviour in the dependence on $B$ also for more complicated 4 -spin operators like $C_{4}$. We use same parameters as for the Fig. 5 in the main text: $\left(\epsilon_{\mathrm{d}} / \epsilon_{\mathrm{ph}}\right)^{2}=2.5, J=1, \Delta=0.8, \omega=1.6 \omega_{\mathrm{ph}}$, $\omega_{\mathrm{ph}}=T_{\mathrm{ph}}=1, N=12$

Code availability. Custom computer codes used in this study are available from the corresponding author upon request. Documentation of the codes is not available.

Data availability. Data is available from the corresponding author upon reasonable request.

\section{References}

1. Kikkawa, J. M. \& Awschalom, D. D. Resonant spin amplification in $n$-type GaAs. Phys. Rev. Lett. 80, 4313-4316 (1998).

2. Klaers, J., Schmitt, J., Vewinger, F. \& Weitz, M. Bose-Einstein condensation of photons in an optical microcavity. Nature 468, 545-548 (2010).

3. Demokritov, S. et al. Bose-Einstein condensation of quasi-equilibrium magnons at room temperature under pumping. Nature 443, 430-433 (2006).

4. Kasprzak, J. et al. Bose-Einstein condensation of exciton polaritons. Nature 443, 409-414 (2006).

5. Allen, P. B. Theory of thermal relaxation of electrons in metals. Phys. Rev. Lett. 59, 1460-1463 (1987).

6. Faddeev, L. Algebraic aspects of the Bethe ansatz. Int. J. Mod. Phys. A 10, 1845-1878 (1995).

7. Grabowski, M. P. \& Mathieu, P. Structure of the conservation laws in integrable spin chains with short range interactions. Ann. Phys. 243, 299-371 (1995).

8. Prosen, T. \& Ilievski, E. Families of quasilocal conservation laws and quantum spin transport. Phys. Rev. Lett. 111, 057203 (2013).

9. Mierzejewski, M., Prelovšek, P. \& Prosen, T. Identifying local and quasilocal conserved quantities in integrable systems. Phys. Rev. Lett. 114, 140601 (2015).

10. Ilievski, E., Medenjak, M. \& Prosen, T. Quasilocal conserved operators in the isotropic Heisenberg spin-1/2 chain. Phys. Rev. Lett. 115, 120601 (2015).

11. Ilievski, E., Medenjak, M., Prosen, T. \& Zadnik, L. Quasilocal charges in integrable lattice systems. J. Stat. Mech.: Theory Exp. 2016, 064008 (2016).

12. Rigol, M., Dunjko, V., Yurovsky, V. \& Olshanii, M. Relaxation in a completely integrable many-body quantum system: an $a b$ initio study of the dynamics of the highly excited states of $1 \mathrm{~d}$ lattice hard-core bosons. Phys. Rev. Lett. 98, 050405 (2007)

13. Pozsgay, B. The generalized Gibbs ensemble for Heisenberg spin chains. J. Stat. Mech.: Theory Exp. 2013, P07003 (2013).

14. Fagotti, M. \& Essler, F. H. Stationary behaviour of observables after a quantum quench in the spin-1/2 Heisenberg XXZ chain. J. Stat. Mech.: Theory Exp. 2013, P07012 (2013)

15. Wouters, B. et al. Quenching the anisotropic Heisenberg chain: exact solution and generalized Gibbs ensemble predictions. Phys. Rev. Lett. 113, 117202 (2014).

16. Pozsgay, B. et al. Correlations after quantum quenches in the $X X Z$ spin chain: Failure of the generalized Gibbs ensemble. Phys. Rev. Lett. 113, 117203 (2014).

17. Ilievski, E. et al. Complete generalized Gibbs ensembles in an interacting theory. Phys. Rev. Lett. 115, 157201 (2015).

18. Vidmar, L. \& Rigol, M. Generalized Gibbs ensemble in integrable lattice models. J. Stat. Mech.: Theory Exp. 2016, 064007 (2016).

19. Essler, F. H. L. \& Fagotti, M. Quench dynamics and relaxation in isolated integrable quantum spin chains. J. Stat. Mech.: Theory Exp. 2016, 064002 (2016).

20. Ilievski, E., Quinn, E. \& Caux, J.-S. From interacting particles to equilibrium statistical ensembles. Phys. Rev. B 95, 115128 (2017).

21. De Luca, A., Collura, M. \& De Nardis, J. Non-equilibrium spin transport in the XXZ chain: steady spin currents and emergence of magnetic domains. Preprint at https://arxiv.org/abs/1612.07265 (2016)

22. Langen, T. et al. Experimental observation of a generalized Gibbs ensemble. Science 348, 207-211 (2015).

23. Mourigal, M. et al. Fractional spinon excitations in the quantum Heisenberg antiferromagnetic chain. Nat. Phys. 9, 435-441 (2013).

24. Jung, P., Helmes, R. W. \& Rosch, A. Transport in almost integrable models: Perturbed Heisenberg chains. Phys. Rev. Lett. 96, 067202 (2006).

25. Jung, P. \& Rosch, A. Spin conductivity in almost integrable spin chains. Phys. Rev. $B$ 76, 245108 (2007).
26. Jung, P. \& Rosch, A. Lower bounds for the conductivities of correlated quantum systems. Phys. Rev. B 75, 245104 (2007).

27. Bertini, B., Essler, F. H. L., Groha, S. \& Robinson, N. J. Prethermalization and thermalization in models with weak integrability breaking. Phys. Rev. Lett. 115, 180601 (2015).

28. De Luca, A. \& Rosso, A. Dynamic nuclear polarization and the paradox of quantum thermalization. Phys. Rev. Lett. 115, 080401 (2015).

29. De Luca, A., Rodrguez-Arias, I., Müller, M. \& Rosso, A. Thermalization and many-body localization in systems under dynamic nuclear polarization. Phys. Rev. B 94, 014203 (2016).

30. Cirac, J. I., Blatt, R., Zoller, P. \& Phillips, W. D. Laser cooling of trapped ions in a standing wave. Phys. Rev. A 46, 2668-2681 (1992).

31. Benatti, F., Nagy, A. \& Narnhofer, H. Asymptotic entanglement and Lindblad dynamics: a perturbative approach. J. Phys. A: Math. Theor. 44, 155303 (2011).

32. Li, A. C. Y., Petruccione, F. \& Koch, J. Perturbative approach to Markovian open quantum systems. Sci. Rep. 4, 4887 (2014).

33. Petruccione, F. \& Breuer, H.-P. The Theory of Open Quantum Systems (Oxford Univ. Press, 2002).

34. Affleck, I. \& Oshikawa, M. Field-induced gap in Cu benzoate and other $s=(1) /(2)$ antiferromagnetic chains. Phys. Rev. B 60, 1038-1056 (1999)

35. Nojiri, H., Ajiro, Y., Asano, T. \& Boucher, J. Magnetic excitation of $s=1 / 2$ antiferromagnetic spin chain $\mathrm{Cu}$ benzoate in high magnetic fields. New J. Phys 8, 218 (2006).

36. Kimura, S. et al. Collapse of magnetic order of the quasi one-dimensional ising-like antiferromagnet $\mathrm{BaCo}_{2} \mathrm{~V}_{2} \mathrm{O}_{8}$ in transverse fields. J. Phys. Soc. Jpn 82, 033706 (2013).

37. Niesen, S. K. et al. Substitution effects on the temperature versus magnetic field phase diagrams of the quasi-one-dimensional effective Ising spin-(1)/(2) chain system $\mathrm{BaCo}_{2} \mathrm{~V}_{2} \mathrm{O}_{8}$. Phys. Rev. B 90, 104419 (2014).

38. Shindou, R. Quantum spin pump in $s=1 / 2$ antiferromagnetic chains-holonomy of phase operators in sine-Gordon theory-. J. Phys. Soc. Jpn 74, 1214-1223 (2005)

39. Genske, M. \& Rosch, A. Floquet-Boltzmann equation for periodically driven Fermi systems. Phys. Rev. A 92, 062108 (2015).

40. D'Alessio, L. \& Rigol, M. Long-time behavior of isolated periodically driven interacting lattice systems. Phys. Rev. X 4, 041048 (2014).

41. Lazarides, A., Das, A. \& Moessner, R. Equilibrium states of generic quantum systems subject to periodic driving. Phys. Rev. E 90, 012110 (2014).

42. Ponte, P., Chandran, A., Papić, Z. \& Abanin, D. A. Periodically driven ergodic and many-body localized quantum systems. Ann. Phys. 353, 196-204 (2015).

43. Lazarides, A., Das, A. \& Moessner, R. Periodic thermodynamics of isolated quantum systems. Phys. Rev. Lett. 112, 150401 (2014).

44. Canovi, E., Kollar, M. \& Eckstein, M. Stroboscopic prethermalization in weakly interacting periodically driven systems. Phys. Rev. E 93, 012130 (2016).

45. Zotos, X., Naef, F. \& Prelovšek, P. Transport and conservation laws. Phys. Rev. B 55, 11029-11032 (1997).

46. Prosen, T. Open $X X Z$ spin chain: nonequilibrium steady state and a strict bound on ballistic transport. Phys. Rev. Lett. 106, 217206 (2011).

47. Mierzejewski, M., Prelovšek, P. \& Prosen, T. Breakdown of the generalized Gibbs ensemble for current-generating quenches. Phys. Rev. Lett. 113, 020602 (2014).

48. Sinova, J., Valenzuela, S. O., Wunderlich, J., Back, C. H. \& Jungwirth, T. Spin hall effects. Rev. Mod. Phys. 87, 1213-1260 (2015).

49. Berges, J., Borsányi, S. \& Wetterich, C. Prethermalization. Phys. Rev. Lett. 93, 142002 (2004).

50. Moeckel, M. \& Kehrein, S. Interaction quench in the hubbard model. Phys. Rev. Lett. 100, 175702 (2008).

51. Kollar, M., Wolf, F. A. \& Eckstein, M. Generalized Gibbs ensemble prediction of prethermalization plateaus and their relation to nonthermal steady states in integrable systems. Phys. Rev. B 84, 054304 (2011).

52. Essler, F. H. L., Kehrein, S., Manmana, S. R. \& Robinson, N. J. Quench dynamics in a model with tuneable integrability breaking. Phys. Rev. B 89, 165104 (2014).

53. Mierzejewski, M., Prosen, T. \& Prelovšek, P. Approximate conservation laws in perturbed integrable lattice models. Phys. Rev. B 92, 195121 (2015).

54. Caux, J.-S. \& Essler, F. H. L. Time evolution of local observables after quenching to an integrable model. Phys. Rev. Lett. 110, 257203 (2013).

55. Caux, J.-S. The quench action. J. Stat. Mech.: Theory Exp. 2016, 064006 (2016)

56. Zotos, X. Finite temperature Drude weight of the one-dimensional spin- 1/2 Heisenberg model. Phys. Rev. Lett. 82, 1764-1767 (1999).

57. Forster, D. Hydrodynamic Fluctuations, Broken Symmetry, and Correlation Functions (Benjamin, 1975).

\section{Acknowledgements}

We acknowledge useful discussions with S. Diehl, F.H.L. Essler, M. Fagotti, E. Ilievski, M. Mierzejewski, J. De Nardis, T. Prosen and M.C. Rudner, H.F. Legg for reading the 
manuscript, and financial support of the German Science Foundation under CRC 1238 (project C04) and CRC TR 183 (project A01).

\section{Author contributions}

A.R. and Z.L. designed the study, Z.L. and F.L. performed analytical calculations and F.L. implemented the numerical codes, all authors analysed the results and contributed to the manuscript.

\section{Additional information}

Competing interests: The authors declare no competing financial interests.

Reprints and permission information is available online at http://npg.nature.com/ reprintsandpermissions/

How to cite this article: Lange, F. et al. Pumping approximately integrable systems. Nat. Commun. 8, 15767 doi: 10.1038/ncomms15767 (2017).
Publisher's note: Springer Nature remains neutral with regard to jurisdictional claims in published maps and institutional affiliations.

(c) (i) Open Access This article is licensed under a Creative Commons Attribution 4.0 International License, which permits use, sharing, adaptation, distribution and reproduction in any medium or format, as long as you give appropriate credit to the original author(s) and the source, provide a link to the Creative Commons license, and indicate if changes were made. The images or other third party material in this article are included in the article's Creative Commons license, unless indicated otherwise in a credit line to the material. If material is not included in the article's Creative Commons license and your intended use is not permitted by statutory regulation or exceeds the permitted use, you will need to obtain permission directly from the copyright holder. To view a copy of this license, visit http://creativecommons.org/ licenses/by/4.0/

(C) The Author(s) 2017 